\title{
Achieving High Transparency in Bilateral Teleoperation Using Stiffness Observer for Passivity Control
}

\author{
Reza Monfaredi \\ Depart. of Mechanical Eng. \\ Amirkabir Univ. of Tech. \\ Tehran, Iran \\ Reza_monfaredi@aut.ac.ir
}

\author{
Kamran Razi \\ Depart. of Electrical Eng. \\ Amirkabir Univ. of Tech. \\ Tehran, Iran \\ Kamranrazi@aut.ac.ir
}

\author{
Saeed Shiri Ghydari \\ Depart. of Computer Eng. \\ Amirkabir Univ. of Tech. \\ Tehran, Iran \\ shiry@ce.aut.ac.ir
}

\author{
Seied Mehdi Rezaei \\ Depart. of Mechanical Eng. \\ Amirkabir Univ. of Tech. \\ Tehran, Iran \\ Smrezaei@cic.aut.ac.ir
}

\begin{abstract}
In this paper a new approach is developed that increases transparency in bilateral teleoperation. We define a stiffness observer which detects hard contact instant by analyzing the generated force in the slave side to activate a passivity controller. This approach uses passivity based idea of monitoring energies flowing in and out of the teleoperator. It improves performance of previous works by eliminating instability in transition to hard contact. It also provides a more general and less conservative approach to passivity control of teleoperated systems. The performance of the new approach has been investigated through simulation results.
\end{abstract}

Index Terms -teleoperation, transparency, passivity control, force feedback, master-salve robot

\section{INTRODUCTION}

Some robotic applications require human intelligence to operate well in the remote environment. The most powerful tool for these applications is a master-slave robot. In this system the remote robot (slave) is being manipulated in real time through the master robot by human operator who takes visual feedback from the slave robot.

Recent researches in this field focus on adding improved force feedback to the system for creating more transparent interface for human operator (bilateral teleoperator)[1][2],[3],[4].

In bilateral teleoperation, the contact forces affecting the slave are fed back to the master to give the operator a feeling of the actual operation. The term transparency is used to describe how close this feeling is to the feeling when performing the same task manually without the teleoperator [4]. It is desirable to build and control the teleoperator such that the transparency is as high as possible. However, there is a trade off between transparency and stability of bilateral teleoperators. Since transparency must often be reduced in order to guarantee stable operation in wide range of environment impedances.

In typical control methods for teleoperated systems the dynamic models of operator and environment must be involved in the system design, however the environment and operator have nonlinear and unknown dynamic models that can not be easily extracted.

One promising approach is the use of passivity to guarantee stable operation without exact knowledge of model information [5].
Anderson and Spong [5] have used the passivity concept for telerobotic systems with time delay. They used passivity to design a system that is passive for all operating conditions. It is known that this method is over conservative and reduces the system transparency [3], [6]. Hannaford and Rya [3] developed a passivity controller that controls the teleoperation system's passivity, using a passivity observer (P.O.) and a passivity controller (P.C.). By application of passivity control, the stability and transparency can be treated separately, without affecting each other, so the performance of the system can be improved by increasing transparency.

Passivity controller reduces the transparency by affecting the force/velocity signals. This can be solved by limiting passivity control to situation when the teleoperation goes to instability.

In this paper it is shown that the passivity observer introduced in [3] and [6] is a conservative method to detect instability, because passivity is sufficient condition for stability[2],[6]. It is not a necessary condition. This has the effect that in some cases of free motion and soft contact, the system might be stable while the passivity observer shows negative value. In the other hand, in some cases when a hard contact occurs, the passivity observer cannot turn on the passivity controller immediately until the internal energy becomes negative. This will reduce transparency of the system and will cause instability in transition to the hard contact. Analyzing these cases for a typical teleoperation system as well as the system is developed in this paper. It is shown that P.O./P.C. approach isn't applicable for all teleoperation systems in general as well as the system developed in this paper with specific dynamic. So a new approach should be developed to control passivity in wide Varity of teleoperation systems.

The hard contact in presence of time delay is a main cause of instability in teleoperated systems [2],[3],[4],[5]. Instability could be eliminated by detection of hard contact and immediate application of passivity controller after contact.

In this paper passivity observer is substituted with a stiffness observer which analyses the generated force in slave side to detect when the slave robot contacts to the hard environment. The stiffness observer detects hard contact by estimating stiffness and turns the passivity controller on when hard contact occurs. 


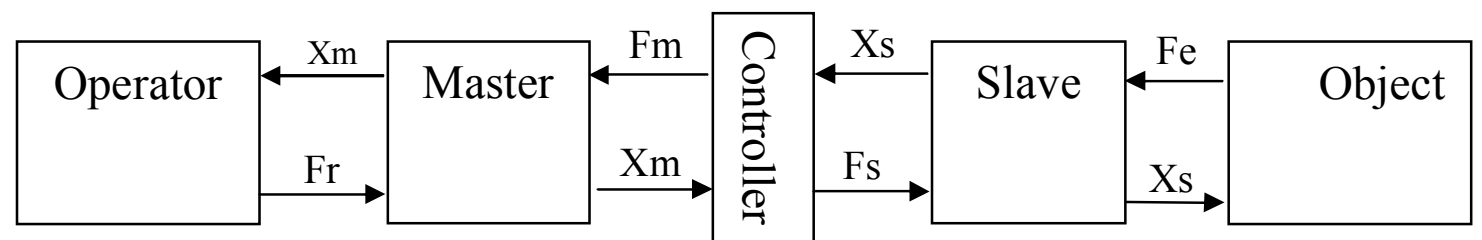

Fig. 1 The general model of a teleoperation system

TABLE I

DIFFERENTIAL EQUATION OF TELEOPERATION COMPONENTS

\begin{tabular}{|l|l|}
\hline \multicolumn{1}{|c|}{ name } & \multicolumn{1}{c|}{ Differential equation } \\
\hline 1) Human & $\ddot{X}_{m} M_{h}+\left(\dot{X}_{h}-\dot{X}_{m}\right) B_{h}+\left(X_{h}-X_{m}\right) K_{h}=F_{r}$ \\
\hline 2) master & $J_{m} \ddot{\theta}_{m}=F_{r} L_{m}-T_{a m}$ \\
\hline \multirow{2}{*}{ 3) controller } & $T_{a m}=P I D_{m} \cdot\left[T_{a m}(t)-T_{a s}(t-T)\right] G_{m-m o t o r}{ }^{a}$ \\
\cline { 2 - 3 } & $T_{a s}=P I D_{s} \cdot\left[\theta_{s}(t)-\theta_{m}(t-T)\right] G_{s-m o t o r}$ \\
\hline 4) Salve & $J_{S} \ddot{\theta}_{S}=T_{a s}(t)-F_{e} L_{s}$ \\
\hline 5) Environment & $L_{S} B_{e} \dot{\theta}_{S}+L_{S} k_{e} \theta_{S}=F_{e}$ \\
\hline
\end{tabular}

${ }^{\mathrm{a}} \mathrm{T}$ is the time delay of signals

The performance of this method has been investigated through simulation results.

\section{TELEOPERATOR STRUCTURE}

Teleoperation systems involve five distinct sections, 1) human, 2) master robot, 3) controller, 4) salve robot and 5) environment [7]. Fig. 1 shows schematic of a typical master-salve system.[1]

There are five architectures for the controller design.[4]

\section{1) Position-position (position error) \\ 2) Force-position (force-velocity) \\ 3) Position - force (velocity-position) \\ 4) Force - force \\ 5) Four channel controller}

We use the second architecture, Force-position, for

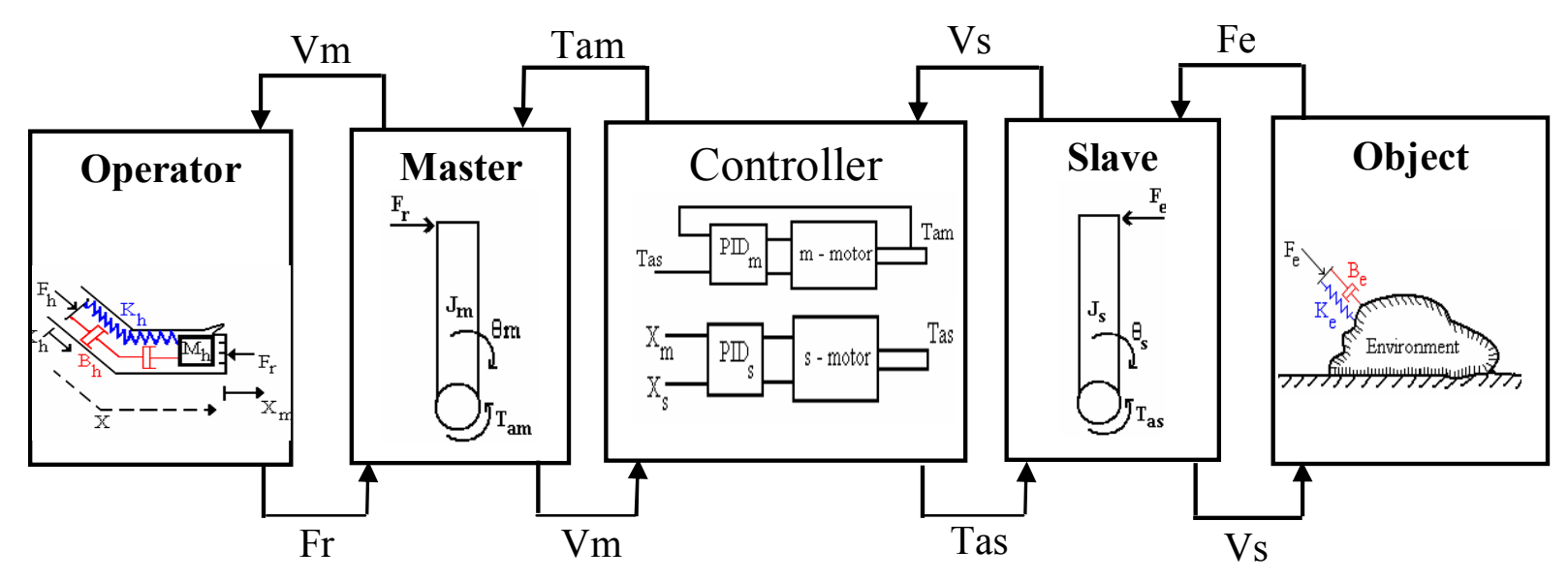

Fig. 2 The teleoperation system model

our controller design, in which the position/velocity signal is fed forward from master robot to the slave robot and the force signal is fed backward form salve robot to the master robot.

\section{TELEOPERATION SYSTEM MODELING}

Yokokohji [8] discusses a model in which the dynamics of the human arm/hand are assumed to be a simple spring, damper and mass system. The environment is modeled as a simple damper-spring system. We modeled our system's operator and environment respectively as a mass_damper_spring and damper_spring system. The differential equation of each part of teleoperation system of one degree of freedom is arranged in Table I. Where $J_{m}$ and $J_{s}$ are the master and slave rotational inertia, $\theta_{m}$ and $\theta_{s}$ are the angular position of master and slave robots 


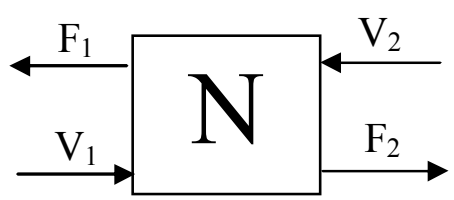

Fig. 3 A two-port network system

respectively, $T_{a m}$ and $T_{a s}$ are the torque generated by master and slave motors. $G_{m}$ and $G_{s}$ are the master robot's motor and save robot's motor transfer function (containing mechanical and electrical constant of motors), respectively. $K_{e}$ and $B_{e}$ represent environment characteristic. And finally, $X_{h}$ represents human operator displacement.

Fig. 2 shows the model of teleoperation system that is used in this paper.

In the following section the passivity theorem and passivity controller are briefly explained. We then analyzed the passivity concept for a typical teleoperation system as well as our supposed system with specific dynamic to show that P.O./P.C. is not a general controller to be applied to all teleoperation systems. Then in section 5 we developed a new observer to detect hard contact as a measure for instability initiation and activate the passivity controller after instability detection.

\section{IV- PASSIVITY THEORY}

Suppose a two-port network system, N, such as shown in Fig. 3.

Now the passivity concept could be defined as following:

\section{A. Passivity Definition}

A two-port network system, with initial energy storage $\mathrm{E}(0)$ is passive if and only if:

$\int\left[F_{1}(t) V_{1}(t)+F_{2}(t) V_{2}(t)\right](d t)+E(0) \geq 0$

$\forall t \geq 0$

The sign convention for all forces and velocities is defined so that their product is positive when power enters the system port. [9], [10], [11]0.

In following we show that passivity is only a sufficient condition for stability and we will conclude that P.O./P.C. isn't applicable for all teleoperation systems and it's application domain is limited to some special teleoperation systems.

\section{B. Passivity is a sufficient condition for stability}

It is known that passivity is only sufficient condition for stability[2],[6] and not a necessary condition, It means that if a system is passive then it is

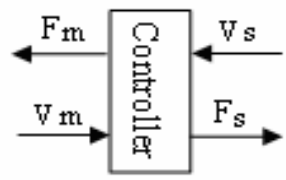

Fig. 4 The controller as a two-port network system
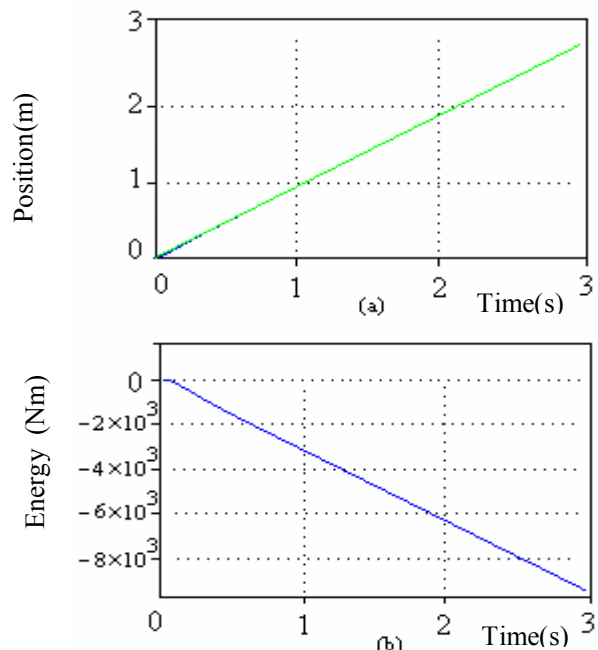

Fig. 5 Free motion a) master and slave position b) system energy stable, but a system that is active isn't necessarily an unstable system.

Indeed, a teleoperation system may go to instability if it losses energy increasingly, so when the internal energy of the system is bounded by finite negative value the system could be stable.

In the next three subsections the internal energy of a typical teleoperation system as well as this research's teleoperated system is discussed. Three possible modes,

1) Free motion, 2) Soft contact and 3) Hard contact are considered.

\section{Passivity in free motion}

Suppose teleoperation controller as a two- port network system as shown in fig. 4 , in the free motion there is no external force in the slave side, so the force applied to the master, $F_{m}$, is to be also zero. In the other hand as the slave arm has to follow the maser position so it requires energy. Integration of $V_{s} F_{s}$ over time results in negative value. In this situation the system is active and stable.

The gravity and friction compensations may change the configuration of the energy flow in and out of the teleoperated systems [6]. In this study only the systems
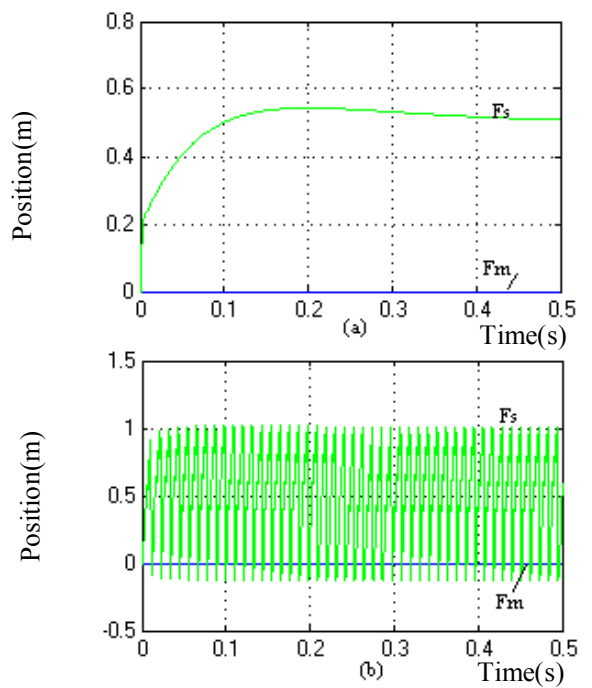

Fig. 6 The master and slave forces in free motion simulation. a) Without passivity controller b) With passivity controller. 

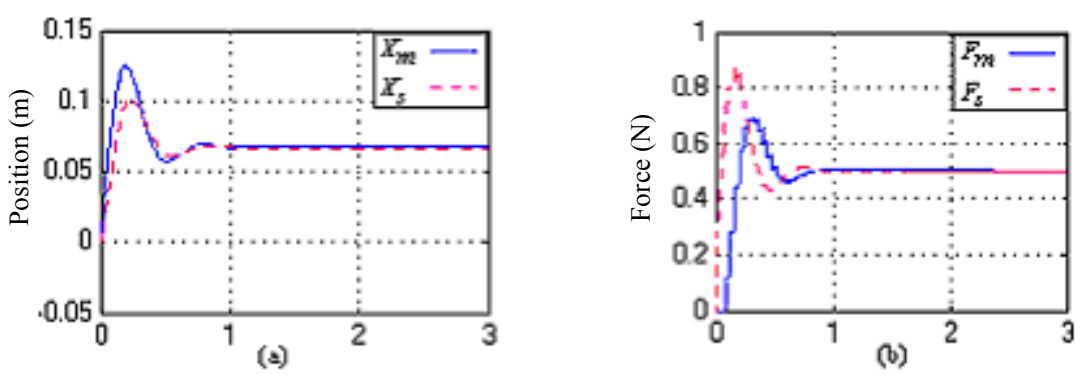

Fig. 8 Master and slave a) positions and b) forces, in soft contact $(k=50 \mathrm{~N} / \mathrm{m})$ simulation, without passivity controller
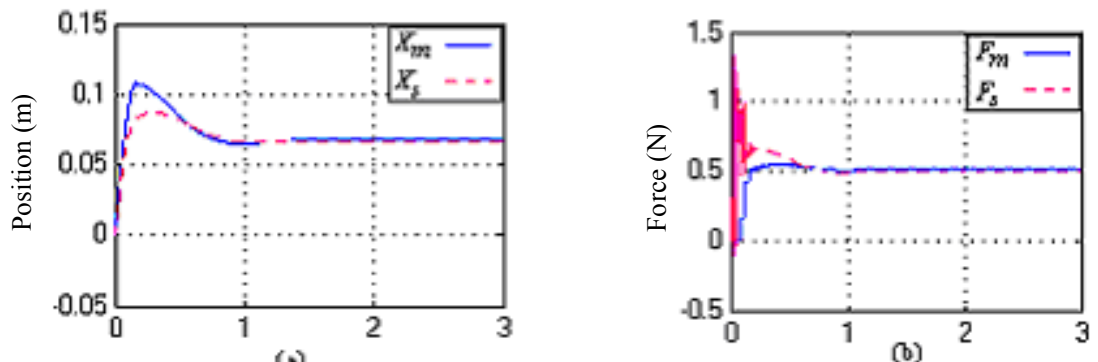

Fig. 9 Master and slave a) positions and b) forces, in soft contact ( $k=50 \mathrm{~N} / \mathrm{m})$ simulation, with passivity controller

which don't have any gravity and friction compensator are discussed.

Fig. 5 (a) shows the master and salve positions in free motion for the system. In this simulation a step torque is applied to the master arm by operator. Fig. 5(b) shows that although the internal energy of system becomes negative, however the system is stable. Turning on the passivity controller in this state will reduce the transparency of the system. Fig. 6 shows simulation results when passivity controller is on. This figure shows that activating passivity controller in free motion results

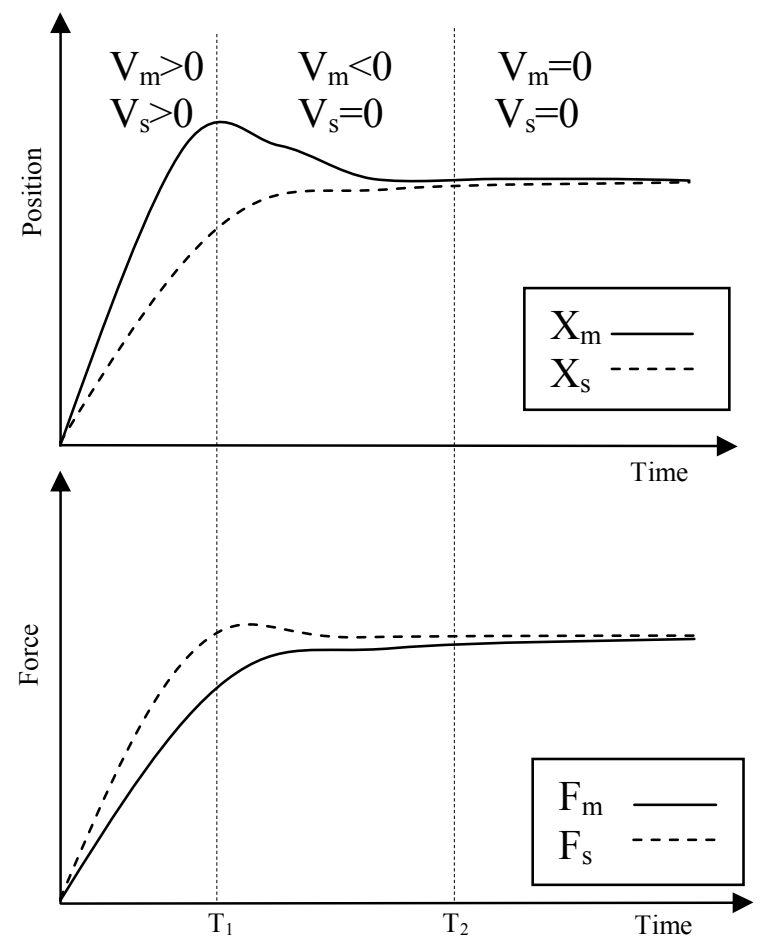

Fig. 7 Soft contact in oscillating salve force that will reduce the system performance.

\section{Passivity in soft contact}

Fig. 7 shows a response of a typical teleoperated system to a step input, in soft contact. In the time interval between $T_{1}$ and $T_{2}$, the value of $V_{s}$ is almost zero, because the slave arm has contacted to external object, however $V_{m}$ is negative. Using (1), we know that the teleoperated system gives energy to the environment and is active, however the system is stable.

Activating the passivity controller in this situation reduces the system transparency.

Simulation shows that in soft contact, the system is stable, but passivity observer shows that the system generates energy, so it isn't passive.

Fig. 8 and Fig. 9 show the positions and forces of the master and slave robot when a soft contact occurs, without and with passivity controller, respectively. As shown in fig. 9(b), the passivity controller results in some oscillation and reduces transparency.

The contacts for different environments stiffness are simulated. The results show that passivity controller activation is useful only for environment stiffness greater than a specific value. This stiffness value certainly depends on the teleoperation system dynamic.

Simulation of supposed system in presence of 40 ms time delay shows that our systems needs to be stabilized by passivity controller for stinffness greater than $400 \mathrm{~N} / \mathrm{m}$.

\section{E. Passivity in hard contact}

Previous experiments[3],[6], show that in some cases, the passivity observer cannot turn on the passivity controller immediately until the internal energy becomes negative, resulting delay in stabilizing the system, and force bounces such as force bounces reported by Hannaford [3]. 


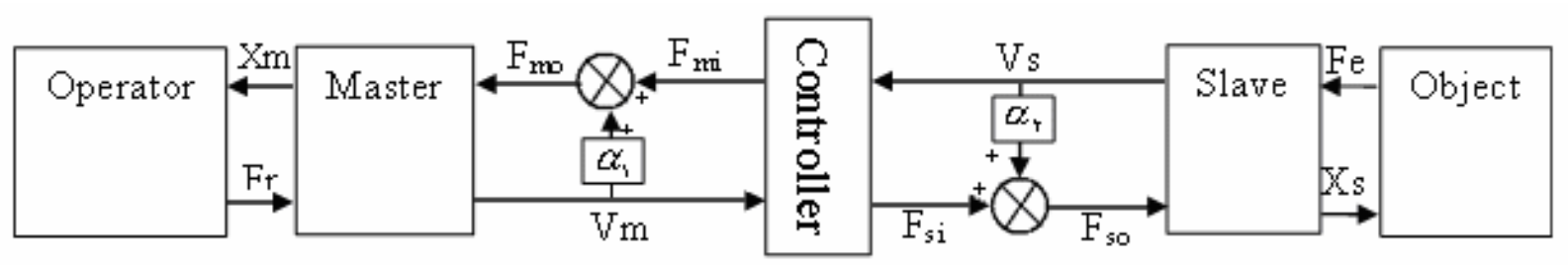

Fig. 10 A passivity controller

Passivity control is a conservative approach that guarantees the system's stability by making system passive in all of situations.

In general, passivity observer is not a suitable observer to detect instability, because as is shown it is probable for internal energy to become negative in free motion and soft contact. Passivity observer also could cause problem in hard contact.

In next section passivity observer is substituted with a stiffness observer. This observer analyses the force generated in the slave side to detect when the slave robot contacts to the hard environment for identifying the system instability time. It should be not that hard contact could be defined as different values for environment corresponding to operation system dynamic characteristics.

\section{STIFFNESS OBSERVER}

Pervious experiments [3], [4] show that a system with time delay goes to instability when contacting to a hard environment. As mentioned before our system gets unstable for stiffness values greater than $400 \mathrm{~N} / \mathrm{m}$. so hard contact could be defined as $k \geq 400 \mathrm{~N} / \mathrm{m}$. If a stiffness observer is used to detect hard contact instant and then the passivity controller is turned on, the system stability for any desired stiffness is guarantied.

Using environment model shown in TABLE I, the stiffness observer equation could be derived as Follows:

$$
\begin{aligned}
& L_{S} B_{e} \dot{\theta}_{S}+L_{S} \cdot k_{e} \theta_{S}=F_{e} \\
& K_{e}=\frac{F_{e}-B_{e} L_{s} \dot{\theta}_{S}}{L_{S} \theta_{S}}
\end{aligned}
$$

RLS method could be used to identify $k_{e}$ and $b_{e}$ values but using RLS method increase the system time delay and it could result in instability. It is known that a hard environment has no damping characteristics, so $B_{e}$ could be eliminated in (2) for simplification. This simplification makes the observer to be conservative, because it estimates the stiffness of the external object more than what it is. The simplified equation can be written as follows:

$$
K_{e}=\frac{F_{e}}{L_{S} \theta_{S}}
$$

Where $\theta_{S}$ is measured from the contact time.

This stiffness observer detects hard contacts, if the threshold is set to $400 \mathrm{~N} / \mathrm{m}$, then the stiffness observer could be able to detect hard contact, i.e. when $K_{e} \geq 400 \mathrm{~N} / \mathrm{m}$. When the hard contact is detected, the passivity controller is turned on to stabilize the system.

Using impedance causality approach, and

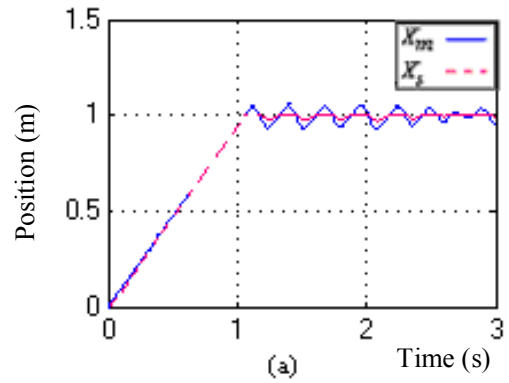

Fig. 11 Contact with a hard environment $(5000 \mathrm{~N} / \mathrm{m})$, a) the master and slave position b) the master and slave motor torque, c) system energy, without passivity controller.

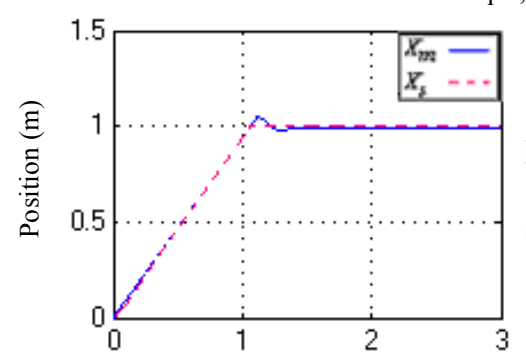

(a)

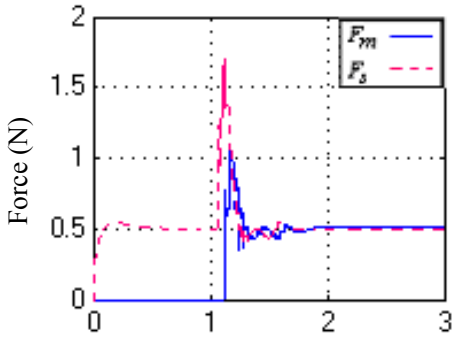

(b)

Time (s)
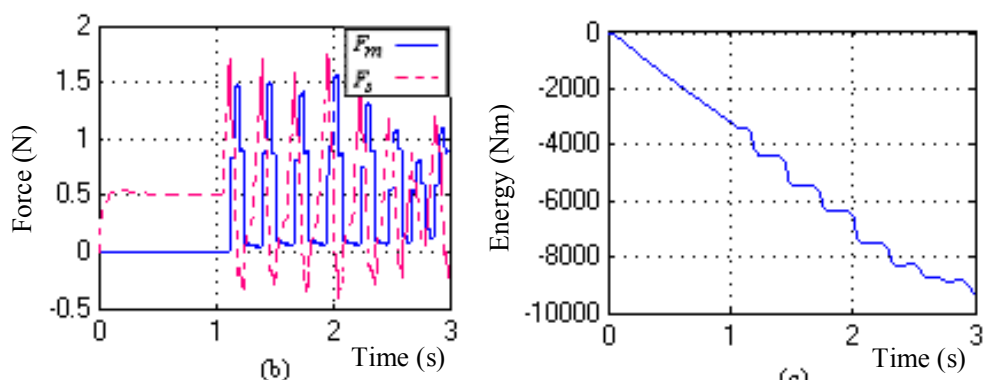

(c)

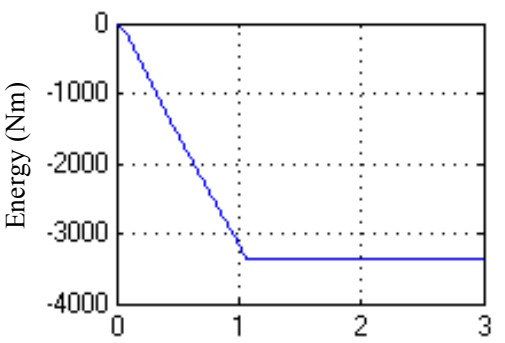

(c) Time (s)

Fig. 12 Contact with a hard environment $(5000 \mathrm{~N} / \mathrm{m})$, a) the master and slave position $\mathrm{b})$ the master and slave motor torque, c) system energy, with passivity controller. 
supposing that the operator and environment are passive in nature [8],[5], the passivity controller is implemented by adding $\alpha_{1}$ and $\alpha_{2}$ to the teleoperation model as shown in Fig. 10. For more details about $\alpha_{1}$ and $\alpha_{2}$ estimation see [3].

\section{SIMULATION RESULTS}

Simulation is done using the Simulink/Matlab environment. A step torque is applied to the master arm $\left(\mathrm{F}_{\mathrm{h}}=0.5 \mathrm{Nm}\right) . k_{e}$ is set to $5000(\mathrm{~N} / \mathrm{m})$ in $\mathrm{t}=1 \mathrm{~s}$. The system is supposed to have a time delay of $40 \mathrm{~ms}$. Fig. 11 shows that the system goes to instability when hard contact occurs $(\mathrm{k}>=400 \mathrm{~N} / \mathrm{m})$.

Then the stiffness observer is applied to detect hard contact instant and the passivity controller is activated when hard contact is occurred. As Fig. 12 shows, this eliminates oscillations and stabilizes the teleoperated system.

Note that only one force bounce is produced in hard contact, as shown in fig. 12(b).

The stiffness observer improves the teleoperated system transparency. It also provides more general way for detecting instability. As discussed in section V, the teleoperated systems generally have negative internal energy in free motion and soft contact, so transition from positive internal energy to negative internal energy, could not be used as a measure of system instability.

\section{CONCLUSION}

In this paper a new approach is developed that increases the system transparency. Passivity observer is substituted with a stiffness observer which analyses the force generated in slave side to detect when the slave robot contacts to hard environment. The stiffness observer detects hard contact by estimating stiffness and turns the passivity controller on when hard contact occurs.

It is discussed that passivity observer is a conservative observer for instability detection, because passivity is sufficient condition for stability[2] and it is not a necessary condition. This has the effect that in some cases of free motion and soft contact, the system might be stable while the passivity observer shows negative value and turns on the passivity controller to reduce the system transparency.
Simulation results show that using stiffness observer increases the system transparency by eliminating force bounces that are reported in pervious works.

To estimate stiffness, simplification was done by neglecting the damping ratio in stiffness estimation that may cause the observer to act conservatively. In future, the research will be concentrated on developing new observer such as impedance observer, chattering observer and also a learning method to activate the passivity controller and to reduce conservatism in instability detection.

\section{REFERENCES}

[1] Frisoli, E. Sotgiu and checcacci, "Theoretical and experimental evaluation of a 2-channal bilateral force reflection teleoperation system. 2002.

[2] D. Lawrence, A., "Stability and transparency in bilateral teleoperation," IEEE Transactions on robotics and automation, October 1993, Vol. 9, No. 5, pp. 624-637.

[3] B. Hannaford, J. Rya, and D. Kwon, "Stable teleoperation with time domain passivity control," Proc. IEEE Int. Conf. Robotics and Automation, 2002

[4] H. Flemmer, "Control design and performance analysis of force reflective teleoperators- passivity based approach", doctoral thesis, Department of machine design, Royal institute of technology, 2004.

[5] R. J. Anderson and M. W. Spong, "asymptotic stability for force reflecting teleoperators with time delay," Int, J. Robot. Res., vol.11, no. 2, pp. 135-149, 1992.

[6] B. Hannaford and H. Rya, "Time Domain passivity control of haptic interfaces, " IEEE trans. Robotics and Automation,Vol. 18, No. 1, pp. 1-10, 2002.

[7] H. Flemmer, B. Eriksson and J. Wikander, "Passivity issues in bilateral teleoperation- a phase property", doctoral thesis, Department of machine design, Royal institute of technology, 2004.

[8] Y. Yokokohji, T. Yoshikawa, "Bilateral control of master and slave manipulators for ideal kinesthetic coupling-Formulation and experiment," IEEE transaction on robotics and automation, Vol. 10, No 5, October 1994, pp. 605-620.

[9] R. J. Adams and B. Hannaford, "Stable haptic interaction with virtual environments," IEEE tran.. Robot. Automat., Vol 15, pp. $465-474$

[10] C. A. Desoer and M. Vidyasagar," Feedback systems: Inputoutput properties," New York: Academic, 1975

[11] A. J. van der schaft, "L2-Gain and passivity techniques in nonlinear control", ser. Communications and control engineering series. New york: springer, 2000.

[12]J. C. Willems, "Dissipative dynamical systems-Part I: General theory," Arch. Rat. Mech. An.,vol. 45, pp.321-351,1972. 
